\title{
Cellulosic biobutanol by clostridia: challenges and improvements
}

\begin{abstract}
The gradual shift of transportation fuels from oil based fuels to alternative fuel resources and worldwide demand for energy has been the impetus for research to produce alcohol biofuels from renewable resources which focus on utilizing simple sugars from lignocellulosic biomass, the largest known renewable carbohydrate source as an alternative. Currently, the usage of bioethanol and biodiesel do not cover an increasing demand for biofuels. Hence, there is an extensive need for advanced biofuels with superior fuel properties. Biobutanol is regarded to be an excellent biofuel compared to bioethanol in terms of energy density and hygroscopicity, could be produced through acetone-butanol-ethanol (ABE) fermentation process. Even though the ABE fermentation is one of the oldest large-scale fermentation processes, biobutanol yield by anaerobic fermentation remains sub-optimal. For sustainable industrial scale of biobutanol production, a number of obstacles need to be addressed including choice of feedstock, low product yield, product toxicity to strain, multiple endproducts and downstream processing of alcohol mixtures plus the metabolic engineering for improvement of fermentation process and products. Studies on the kinetic and physiological models for fermentation using lignocellulosic biomass provide useful information for process optimization. Simultaneous saccharification and fermentation (SSF) with in-situ product removal techniques have been developed to improve production economics due to the lower biobutanol yield in the fermentation broth. The present review is attempting to provide an overall outlook on the discoveries and strategies that are being developed for biobutanol production from lignocellulosic biomass.
\end{abstract}

Keyword: Lignocellulosic biomass; Biobutanol; Biofuel; Consolidated bioprocessing; Simultaneous saccharification and fermentation; Genetic engineering; Clostridium 